\title{
29. Positive Immunostaining with Dystrophin Antibodies in mdx Skeletal Muscle
}

\author{
By Hikaru TANaKA, ${ }^{*)}$ Kensuke HaYASHI, ${ }^{* *)}$ and Eijiro OZAWA**),***) \\ (Communicated by Setsuro EBASHI, M. J. A., Oct. 14, 1991)
}

\begin{abstract}
The $m d x$ mouse has X-chromosome linked myopathy due to dystrophin deficiency, and is therefore used as a model of human Duchenne muscular dystrophy. As the $m d x$ mouse bears a point mutation resulting in a stop codon in the middle of the dystrophin gene, most of its muscle fibers are not stained in immunohistochemistry with anti-dystrophin antisera. However, a clear immunostaining occurs on the cell membrane in a small number of fibers. We characterized these rare positive fibers in the present study with five different antibodies raised against various regions of dystrophin. They were stained with four of them inluding those raised against the $\mathrm{N}$ - and C-terminals of dystrophin. However, they were not stained with antibody Dy4/6D3 raised against the middle region of dystrophin close to the site corresponding to the mdx mutation. The staining could not be explained by the expression of the dystrophin related protein, an autosomal homologue of dystrophin. We tentatively concluded that the positive staining is due to truncated dystrophin lacking the region corresponding to the mdx mutation.
\end{abstract}

Key words: Dystrophin; $m d x$ mouse; immunohistochemistry.

Introduction. Dystrophin is the protein product of the X-linked Duchenne muscular dystrophy (DMD) locus which is disrupted to yield the phenotypes of DMD and Becker muscular dystrophy (BMD). Dystrophin can be immunohistochemically detected on the cell membrane in normal skeletal and cardiac muscles, but is absent in muscle from DMD patients (Sugita et al., 1988; Zubrzycka-Gaarn et al., 1988; Arahata et al., 1988; Watkins et al., 1988).

The $m d x$ mouse is an X-chromosome linked myopathic mutant (Bulfield et al., 1984) which is devoid of dystrophin (Hoffman et al., 1987; Arahata et al., 1988), and is therefore widely used as an animal model of human DMD. It has a single base substitution within the dystrophin gene exon which is considered to cause premature termination of the peptide chain (Sicinski et al., 1989).

However, a peculiar immunohistochemical observation was made with skeletal muscle samples from DMD patients; while a great majority of muscle fibers was not stained with monoclonal antibody $\mathrm{A1C}$, raised against a region close to the N-terminal of dystrophin, a small number of fibers was clearly stained on the cell membrane (Shimizu et al., 1988). Similar immunostaining was reported with antibodies against other regions of dystrophin, and also in the $m d x$ mouse (Hoffman et al., 1990; Nicholson 1989a). In the present study, we found that this peculiar immunostaining can be observed in $m d x$ mice with four different antibodies raised against various regions of dystrophin but not with an antibody against the middle region close to the site corresponding to the $m d x$ mutation.

Materials and methods. Muscle samples were epaxial or tibialis muscles from 10-week

*) Present address: Department of Pharmacology, Toho University School of Pharmaceutical Sciences, Funabashi, Chiba 274, Japan.

**) Division of Cell Biology, National Institute of Neuroscience, NCNP, Kodaira, Tokyo 187, Japan.

***) To whom correspondence should be addressed. 
old wild type (C57BL/ScSn) and $m d x(\mathrm{C} 57 \mathrm{BL} / \mathrm{ScSn}-m d x)$ mice.

Polyclonal antibodies used were against four different regions of dystrophin raised against synthetic peptides corresponding to amino-acid sequences of human dystrophin deduced from cDNA (Koenig et al., 1988). POOa (Tanaka et al., 1989a), PO4d (Hagiwara et al., 1988), P23b (Yoshida et al., 1990) and P34c (Tanaka et al., 1989a) were raised against residues 11 to 60,440 to 489,2360 to 2409 and 3495 to 3544, respectively. Monoclonal antibody Dy4/6D3 (Nicholson et al., 1989b), raised against a fusion protein containing a 30 $\mathrm{kD}$ fragment of mouse dystrophin corresponding to residues 1180 to 1388 of human cDNA (Koenig et al., 1988), was a gift from Prof. L. V. B. Nicholson. The regions recognized by these antibodies were illustrated in Fig. 1. PDRP, a polyclonal antibody against the dystrophin-related protein (DRP) was prepared and characterized in our previous study (Tanaka et al., 1991).

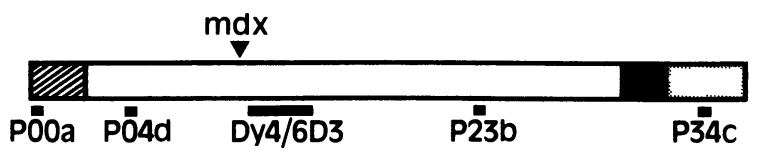

Fig. 1. A schematic of dystrophin with its four domains; N-terminal (striped), rod (open), cystein rich (closed) and C-terminal (stippled; Koenig et al., 1988). The locations of the antigenic peptide for the antibodies are indicated together with the site corresponding to the $m d x$ mutation.

Immunohistochemistry was performed as previously described (Hagiwara et al., 1989). Briefly, $6 \mu \mathrm{m}$ cryosections of muscle tissues were fixed in cold acetone and incubated with the antibodies. They were further incubated with either fluorescein isothiocyanatelabeled goat $\mathrm{F}\left(\mathrm{ab}^{\prime}\right) 2$ anti-rabbit or anti-mouse IgG (TAGO, Inc), and observed with a Nikon FX epi-fluorescent microscope.

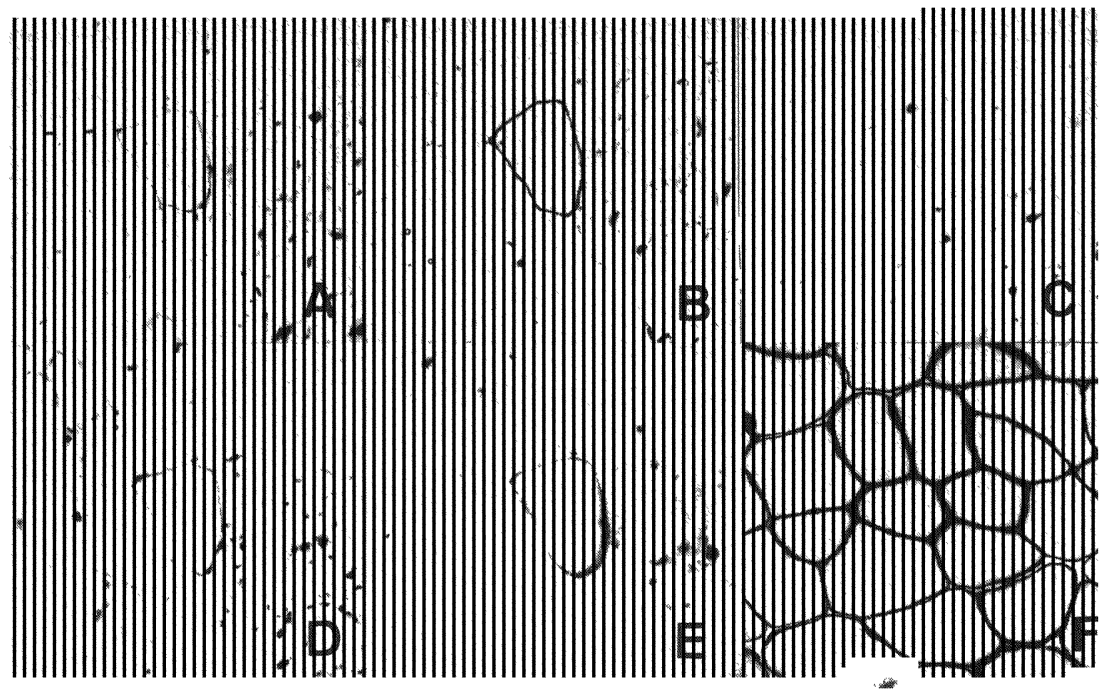

Fig. 2. Frozen sections of skeletal muscles of $m d x$ (A-E: serial sections) and wild type (F) muscles were stained with POOa (A), PO4d (B), Dy4/6D3 (C, F), P23b (D) and $\mathrm{P} 34 \mathrm{c}(\mathrm{E})$ by the indirect fluorescence method. The fiber positively stained by POO, P04d, P23b and P34c was not stained by Dy4/6D3 (C). 
Results. A great majority of the fibers in $m d x$ muscle was not stained with any of the anti-dystrophin antibodies used. Rare positive fibers clearly stained on the cell membrane were observed with $\mathrm{POOa}$, an antibody against the $\mathrm{N}$-terminal of dystrophin. The same

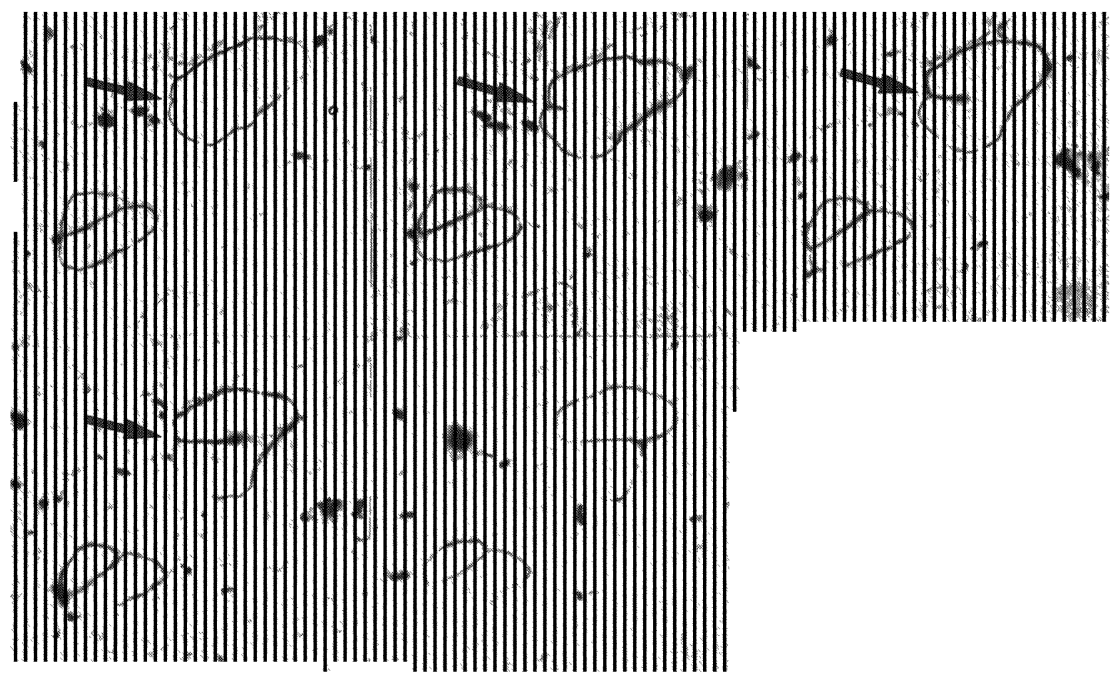

Fig. 3. Serial frozen sections of $m d x$ muscles were stained with PO4d by the indirect fluorescence method. Muscle fiber splitting, (arrows) which is characteristic of dystrophic muscles, were observed in the positively stained region.

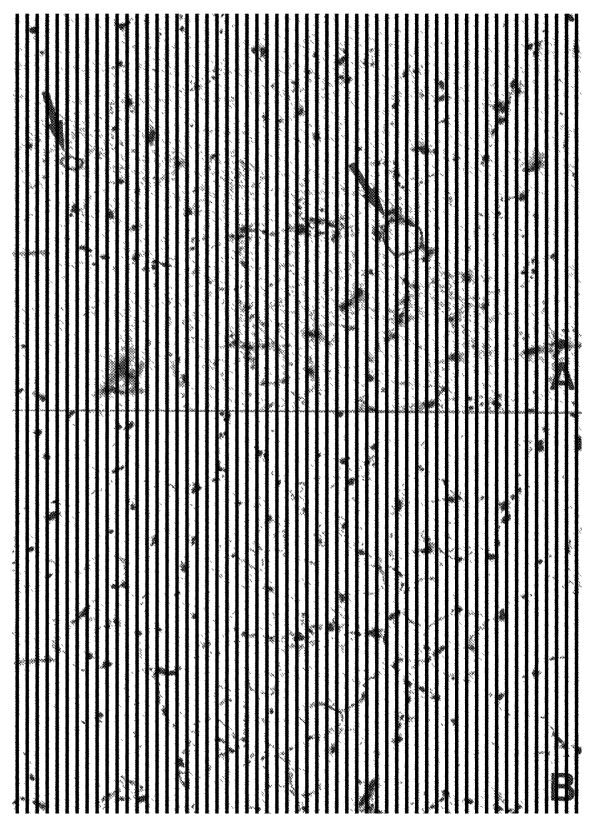

Fig. 4. Serial frozen sections of $m d x$ muscles were stained with PO4d (A) or PDRP (B) by the indirect fluorescence method. The stainings with PO4d (A, arrows), did not coincide with those with PDRP (B). 
fibers were also stained with PO4d and P23b which were against the middle region, and with P34c which was against the C-terminal. However, these fibers were not stained with Dy4/6D3, which was raised against the middle region close to the site corresponding to the $m d x$ mutation and clearly stained the cell membrane in normal mouse muscle (Figs. 1, 2).

Only a few fibers were positively stained in each muscle sample, but they were observed in most samples examined. Staining of serial sections revealed that only a limited area of a given muscle fiber was positively stained. In some cases, muscle fiber splitting, which is characteristic of dystrophic muscles, were observed in the positively stained region (Fig. 3).

This peculiar immunostaining did not correlate with the staining by PDRP. Only a few percent of the $m d x$ muscle fibers were stained with the antibodies against dystrophin while DRP was detected in roughly half of the fibers. Staining of serial sections with PO4d and PDRP clearly demonstrated the distinction between the two types of staining (Fig. 4).

Discussion. The positive fibers in $m d x$ mouse were stained by four different antibodies raised against various regions of dystrophin (Figs. 1, 2), which is consistent with a previous report (Hoffman et al., 1991). Before we conclude that these fibers are indeed "dystrophin positive", we must consider various proteins which might cross-react with dystrophin antibodies. Alpha-actinins and beta-spectrins are known to share sequence similarities with dystrophin in the amino-terminal and rod domains (Hammonds, 1987; Byers et al., 1989). As the positive fibers in $m d x$ muscle were stained with P34c which was raised against the carboxyl-terminal region, the staining can not be explained by the presence of these proteins. The dystrophin-related protein (DRP; Khurana et al., 1990; Tanaka et al., 1991) is the translation product of the autosomal transcript with high homology to the carboxyl-terminal of dystrophin (Love et al., 1989). DRP is expressed on the muscle cell membrane in DMD and BMD patients and $m d x$ mice (Tanaka et al., 1991), and was considered to cross-react with antibodies against the carboxyl-terminal of dystrophin (Tanaka et al., 1989b; 1991). The present results show that the rare positive staining in $m d x$ mice with dystrophin antibody PO4d does not correlate with the staining of DRP (Fig. 4). Bar et al. (1990) have detected a $6.5 \mathrm{k}$ base transcript originating from the dystrophin gene in the liver RNA. As this transcript has no region homologous to the amino-terminal half of the dystrophin cDNA, the corresponding protein, if existed, can not explain the positive immunostaining in $m d x$ mice which was also observed with antibodies POOa and PO4d. From these considerations, we tentatively concluded that the positive immunostaining is due to dystrophin expressed in a small number of muscle fibers.

An important finding made in the present study is that the rare positive fibers were not stained by Dy4/6D3, a monoclonal antibody raised against the middle region close to the site corresponding to the $m d x$ mutation. Hoffman et al. (1990) mentioned that the rare positive fibers were stained with their antibody raised against the same antigenic fusion protein as that used to raise Dy4/6D3; this inconsistency might be due to the polyclonal nature of their antibody. Our results suggest that the truncated dystrophin is expressed in some fibers by some mechanisms to bypass the $m d x$ nonsense mutation. Hoffman et al. (1990) consider that somatic reversion of the $m d x$ mutation itself might be taking place in $m d x$ mice. It is also possible that some kind of "truncation" occurs at the splicing level. In human DMD muscle fibers, quantitative PCR analyses revealed the presence of rare alternatively spliced transcripts lacking exon 2-8 which give rise to "in-framely" truncated transcripts (Chelly et al., 1990). Thus a possible interpretation of the present observations is that leaky alternatively spliced transcripts lacking exons including the mdx mutation site are expressed in some fibers and that they are responsible for the rare dystrophin positive staining in $m d x$ mice. In any case, the mechanisms to bypass the $m d x$ mutation awaits further investigation. 
Acknowledgments. The authors express their thanks to Dr. L. V. B. Nicholson for providing us Dy4/6D3 and to Dr. T. Matsuzaki for providing us $m d x$ mice. This study was partially supported by a Grant (2-1) from the National Center of Neurology and Psychiatry (NCNP) of the Ministry of Health and Welfare Japan, and by Grants from the Ministry of Education, Culture and Science.

\section{References}

Arahata, K. et al.: Nature, 333, 861-863 (1988).

Bar, S., et al.: Biochem. J., 272, 557-560 (1990).

Byers, T. J. et al.: J. Cell Biol., 109, 1633-1641 (1989).

Bulfield, G. et al.: Proc. Natl. Acad. Sci. USA, 81, 1189-1192 (1984).

Chelly, J.: Cell, 63, 1239-1248 (1990).

Hagiwara, Y. et al.: Protoplasma, 151, 11-18 (1989).

Hammonds, R. G. Jr. et al.: Cell, 51, 1 (1987).

Hoffman, E. P. et al.: ibid., 51, 191-928 (1987).

Hoffman, E. P. et al.: J. Neurol. Sci., 99, 9-25 (1990).

Khurana, T. S. et al.: J. Biol. Chem., 265, 16717-16720 (1990).

Koenig, M. et al.: Cell, 53, 219-228 (1988).

Love, D. R. et al.: Nature, 339, 55-58 (1989).

Nicholson, L. V. K. et al.: J. Neurol. Sci., 94, 137-146 (1989a).

Nicholson, L. V. K. et al.: ibid., 94, 126-136 (1989b).

Shimizu, T. et al.: Proc. Japan Acad., 64B, 205-208 (1988).

Sicinski, P. et al.: Science, 244, 1578-1580 (1989).

Sugita, H. et al.: Proc. Japan Acad., 64B, 37-39 (1988).

Tanaka, H. et al.: Protoplasma, 152, 109-111 (1989a).

Tanaka, H. et al.: Proc. Japan Acad., 65B, 238-241 (1989b).

Tanaka, H. et al.: Histochemistry, 96, 1-6 (1991).

Watkins, S. C. et al.: Nature, 333, 863-866 (1988).

Yoshida, M., and Ozawa, E.: J. Biochem., 108, 748-752 (1990).

Zubrzycka-Gaarn, E. E. et al.: Nature, 333, 466-469 (1988). 